Quartic Liénard Equations with Linear Damping

Peer-reviewed author version

HUZAK, Renato (2019) Quartic Liénard Equations with Linear Damping. In:

Qualitative Theory of Dynamical Systems,.

DOI: $10.1007 / \mathrm{s} 12346-018-0302-3$

Handle: http://hdl.handle.net/1942/27452 


\title{
Quartic Liénard equations with linear damping
}

\begin{abstract}
In this paper we prove that the quartic Liénard equation with linear damping $\left\{\dot{x}=y, \dot{y}=-\left(a_{0}+x\right) y-\left(b_{0}+b_{1} x+b_{2} x^{2}+b_{3} x^{3}+x^{4}\right)\right\}$ can have at most two limit cycles, for the parameters kept in a small neighborhood of the origin $\left(a_{0}, b_{0}, b_{1}, b_{2}, b_{3}\right)=(0,0,0,0,0)$. Near the origin in the parameter space, the Liénard equation is of singular type and we use singular perturbation theory and the family blow up. To study the limit cycles globally in the phase space we need a suitable Poincaré-Lyapunov compactification.
\end{abstract}

\section{Introduction}

A simplified version of Hilbert's 16th problem deals with finding the maximum number $l_{n, m}$ of limit cycles of a polynomial Liénard equation

$$
\left\{\begin{array}{l}
\dot{x}=y \\
\dot{y}=-y \sum_{j=0}^{n} a_{j} x^{j}-\sum_{j=0}^{m} b_{j} x^{j}
\end{array}\right.
$$

where $\left(a_{0}, \ldots, a_{n}, b_{0}, \ldots, b_{m}\right) \in \mathbb{R}^{n+m+2}$ and $a_{n}, b_{m} \neq 0$. See e.g. [Sma00, LMT10]. When $m=1$ (resp. $m>1$ ) we call (1) a classical Liénard equation (resp. a generalized Liénard equation). In the classical case, we know that $l_{1,1}=0, l_{2,1}=1$ (see [LdMP77]) and $l_{3,1}=1$ (see [LL12]). In the generalized case, we have $l_{1,2}=1$ (see [Cop89]), $l_{1,3}=1$ (see [DR90, DL96]), $l_{2,2}=1$ (see [DL97]) and $l_{2,3}=3$ (see [WJ02]). As far as we know, only these low degree cases have been completely solved. In our paper we show that $l_{1,4}=2$, under condition that $(1)$ with $(n, m)=(1,4)$ is of singular type. (See the rest of this section for details.)

To prove the uniform finiteness for the polynomial Liénard equations (1) of type $(n, m)$ (i.e., $\left.l_{n, m}<\infty\right)$ one can follow the program formulated in [DH99, Dum06, Rou07]. The program consists of a suitable compactification of the phase space (see [DH99]) and the parameter space (see [Rou07] in the classical case and [Dum06] in the generalized case), for each fixed $(n, m)$, and the determination of all possible limit periodic sets in the Liénard family of the fixed type $(n, m)$.

Using a scaling in the $(x, y, t)$-space we may assume that $a_{n}=1$ for each type $(n, m)$ and $b_{m}= \pm 1$ (resp. $b_{m}=1$ ) for $m \neq 2 n+1$ with $m$ odd (resp. $m$ even). When $m=2 n+1$ then $b_{m} \neq 0$. Using a singular perturbation problem $X_{(A, B)}^{S}:\left\{\dot{x}=y, \dot{y}=-y\left(\sum_{j=0}^{n-1} A_{j} x^{j}+x^{n}\right)-\epsilon\left(\sum_{j=0}^{m-1} B_{j} x^{j} \pm x^{m}\right)\right\}$ and a Hamiltonian perturbation problem $X_{(A, B)}^{H}:\left\{\dot{x}=y, \dot{y}=-\delta y\left(\sum_{j=0}^{n-1} A_{j} x^{j}+x^{n}\right)-\right.$ $\left.\left(\sum_{j=0}^{m-1} B_{j} x^{j} \pm x^{m}\right)\right\}$, with $\delta \sim 0, \delta>0, \epsilon \sim 0, \epsilon>0$ and $\|(A, B)\|=1$, we are 
now able to state the main result of [Dum06]. For a fixed type $(n, m)$, after linear conjugacy and multiplication by a positive constant, all possible phase portraits of $(1)$, with $\left\|\left(a_{0}, \ldots, a_{n-1}, b_{0}, \ldots, b_{m-1}\right)\right\| \neq 0$, can be obtained by studying $X_{(A, B)}^{S}, X_{(A, B)}^{H}$ and (1) with $0<c_{1} \leq\left\|\left(a_{0}, \ldots, a_{n-1}, b_{0}, \ldots, b_{m-1}\right)\right\| \leq c_{2}$, where $c_{1}$ (resp. $c_{2}$ ) is small enough (resp. large enough). Assume that $m<2 n+1$. Then we study $X_{(A, B)}^{H}$ if $\left\|\left(a_{0}, \ldots, a_{n-1}, b_{0}, \ldots, b_{m-1}\right)\right\| \rightarrow 0$ and $X_{(A, B)}^{S}$ if $\left\|\left(a_{0}, \ldots, a_{n-1}, b_{0}, \ldots, b_{m-1}\right)\right\| \rightarrow \infty$. When $m>2 n+1$ then we study $X_{(A, B)}^{S}$ if $\left\|\left(a_{0}, \ldots, a_{n-1}, b_{0}, \ldots, b_{m-1}\right)\right\| \rightarrow 0$ and $X_{(A, B)}^{H}$ if $\left\|\left(a_{0}, \ldots, a_{n-1}, b_{0}, \ldots, b_{m-1}\right)\right\| \rightarrow$ $\infty$. When $m=2 n+1$, then $\epsilon=\left|b_{m}\right|$ and $\delta=\frac{1}{\sqrt{\left|b_{m}\right|}}$. For more details see [Dum06]. When $\left\|\left(a_{0}, \ldots, a_{n-1}, b_{0}, \ldots, b_{m-1}\right)\right\|=0$, then (1) has one singularity $(x, y)=(0,0)$ and no limit cycles (see also [Dum06]).

In this paper, our focus is on the quartic Liénard equation with linear damping

$$
\left\{\begin{array}{l}
\dot{x}=y \\
\dot{y}=-y\left(a_{0}+x\right)-\left(b_{0}+b_{1} x+b_{2} x^{2}+b_{3} x^{3}+x^{4}\right)
\end{array}\right.
$$

where $\left(a_{0}, b_{0}, b_{1}, b_{2}, b_{3}\right) \sim(0,0,0,0,0)$. As explained above, this system is of singular type $(4>2 \cdot 1+1)$ and can be studied by using singular perturbation theory and family blow up. In fact, the regular codimension $m$ nilpotent singularity in Liénard equations of type $(1, m)$ with the parameters kept close to the origin, for $m \geq 4$, is of slow-fast type after a suitable blow-up. When $m=4$, the blow-up is explained in Section 2.1. For $m \geq 4$, the desingularization of the nilpotent singularity is similar and can be found in [Dum06], [DMD11] or [Pan02]. The system $(2)$, near the origin $(x, y)=(0,0)$, is a special case of the so-called regular codimension four saddle-node bifurcations studied in [Huz17]. (When $a_{0}=b_{0}=b_{1}=b_{2}=b_{3}=0$, then (2) has a nilpotent singularity of saddle-node type at the origin.) It will easily follow from [Huz17] that the Liénard equation $(2)$, with $\left(a_{0}, b_{0}, b_{1}, b_{2}, b_{3}\right) \sim(0,0,0,0,0)$, can produce at most two limit cycles in a small $\left(a_{0}, b_{0}, b_{1}, b_{2}, b_{3}\right)$-uniform neighborhood of the origin in the phase space (see Section 2). Due to the length of the paper [Huz17], we decided to prove the "global" version of this result in the present paper.

Theorem 1.1. There exists a small neighborhood $V$ of the origin in the parameter space $\left(a_{0}, b_{0}, b_{1}, b_{2}, b_{3}\right)$ such that (2) has at most two limit cycles for each $\left(a_{0}, b_{0}, b_{1}, b_{2}, b_{3}\right) \in V$.

The proof of Theorem 1.1 consists essentially of two steps. First, using appropriate linear conjugacy and multiplication by positive constant, proposed in [Dum06], we bring (2) to a similar Liénard equation, but of slow-fast type and with the parameters kept on the unit sphere. Then the slow-fast Liénard equation can be studied in arbitrarily large compact sets (resp. near the infinity) in the phase space by using the results of [Huz17] (resp. an appropriate PoincaréLyapunov compactification). It will be clear from the proof that, besides the two limit cycles Hausdorff close to $(x, y)=(0,0)$ found in [Huz17], system (2) has no extra limit cycles. Theorem 1.1 will be proved in Section 2 .

We also study the slow-fast quartic Liénard equation with linear damping

$$
\left\{\begin{array}{l}
\dot{x}=y \\
\dot{y}=-y\left(a_{0}+x\right)-\epsilon\left(b_{0}+b_{1} x+b_{2} x^{2}+b_{3} x^{3}+x^{4}\right)
\end{array}\right.
$$


where $\epsilon \sim 0, \epsilon>0$ and $\left(a_{0}, b_{0}, b_{1}, b_{2}, b_{3}\right) \sim(0,0,0,0,0)$. This slow-fast Liénard equation, near the origin $(x, y)=(0,0)$, is a special case of the well known slow-fast codimension four saddle-node bifurcations studied in [Huz17]. As a simple consequence of Theorem 1.1 in [Huz17], (3) can generate at most two limit cycles in a small $\left(\epsilon, a_{0}, b_{0}, b_{1}, b_{2}, b_{3}\right)$-uniform neighborhood of $(x, y)=(0,0)$ (see Section 3). The following theorem is a global version of this local result:

Theorem 1.2. There exists a small $\epsilon_{0}>0$ and a small neighborhood $V$ of the origin in the parameter space $\left(a_{0}, b_{0}, b_{1}, b_{2}, b_{3}\right)$ such that (3) has at most two limit cycles for each $\left(\epsilon, a_{0}, b_{0}, b_{1}, b_{2}, b_{3}\right) \in\left[0, \epsilon_{0}\right] \times V$.

To prove Theorem 1.2 we use the result of [Huz17], the slow dynamics along the slow curve $\{y=0\}$ of (3) and the same Poincaré-Lyapunov compactification as that used in Section 2. We prove Theorem 1.2 in Section 3.

\section{Proof of Theorem 1.1}

\subsection{Transforming the Liénard equation (2) to a slow-fast system}

We may assume that $a_{0}=0$ in (2). Indeed, after the translation $a_{0}+x \rightarrow x$, (2) becomes

$$
\left\{\begin{array}{l}
\dot{x}=y \\
\dot{y}=-y x-\left(b_{0}+b_{1} x+b_{2} x^{2}+b_{3} x^{3}+x^{4}\right),
\end{array}\right.
$$

with a new parameter $\left(b_{0}, b_{1}, b_{2}, b_{3}\right) \sim(0,0,0,0)$. Now, it suffices to show that there exists a small neighborhood $\widetilde{V}$ of $\left(b_{0}, b_{1}, b_{2}, b_{3}\right)=(0,0,0,0)$ such that $(4)$ has at most two limit cycles for each fixed $\left(b_{0}, b_{1}, b_{2}, b_{3}\right) \in \widetilde{V}$. We denote the system (4) by $X_{b_{0}, b_{1}, b_{2}, b_{3}}$.

Using a linear coordinate change $(x, y)=\left(\epsilon \bar{x}, \epsilon^{2} \bar{y}\right)$, with $\epsilon>0$ and $\epsilon \sim 0$, we convert the system $X_{\epsilon^{4} B_{0}, \epsilon^{3} B_{1}, \epsilon^{2} B_{2}, \epsilon B_{3}}$ to

$$
\left\{\begin{array}{l}
\dot{\bar{x}}=\epsilon \bar{y} \\
\dot{\bar{y}}=\epsilon\left(-\bar{y} \bar{x}-\epsilon\left(B_{0}+B_{1} \bar{x}+B_{2} \bar{x}^{2}+B_{3} \bar{x}^{3}+\bar{x}^{4}\right)\right)
\end{array}\right.
$$

where $\left(B_{0}, B_{1}, B_{2}, B_{3}\right) \in \mathbb{S}^{3}$. After dividing (5) by the positive constant $\epsilon$, we conclude that $X_{\epsilon^{4} B_{0}, \epsilon^{3} B_{1}, \epsilon^{2} B_{2}, \epsilon B_{3}}$ is (linearly) equivalent to

$$
\left\{\begin{array}{l}
\dot{\bar{x}}=\bar{y} \\
\dot{\bar{y}}=-\bar{y} \bar{x}-\epsilon\left(B_{0}+B_{1} \bar{x}+B_{2} \bar{x}^{2}+B_{3} \bar{x}^{3}+\bar{x}^{4}\right),
\end{array}\right.
$$

where $\left(B_{0}, B_{1}, B_{2}, B_{3}\right) \in \mathbb{S}^{3}$. Thus, instead of studying system $X_{\epsilon^{4} B_{0}, \epsilon^{3} B_{1}, \epsilon^{2} B_{2}, \epsilon B_{3}}$, with $\epsilon>0$ and $\left(B_{0}, B_{1}, B_{2}, B_{3}\right) \in \mathbb{S}^{3}$, we can study system (6) which is of singular type. When we vary $\epsilon>0$ and $\left(B_{0}, B_{1}, B_{2}, B_{3}\right) \in \mathbb{S}^{3}$, we completely cover a small neighborhood of $\left(b_{0}, b_{1}, b_{2}, b_{3}\right)=(0,0,0,0)$ (excluding the origin) with $\left(b_{0}, b_{1}, b_{2}, b_{3}\right)=\left(\epsilon^{4} B_{0}, \epsilon^{3} B_{1}, \epsilon^{2} B_{2}, \epsilon B_{3}\right)$. This implies that system $X_{b_{0}, b_{1}, b_{2}, b_{3}}$, with $\left(b_{0}, b_{1}, b_{2}, b_{3}\right) \sim(0,0,0,0)$ and $\left(b_{0}, b_{1}, b_{2}, b_{3}\right) \neq(0,0,0,0)$, can be studied by using singular perturbation problem (6) with $\epsilon>0, \epsilon \sim 0$ and $\left(B_{0}, B_{1}, B_{2}, B_{3}\right) \in \mathbb{S}^{3}$. See also Section 4.2 in [Dum06].

When $\left(b_{0}, b_{1}, b_{2}, b_{3}\right)=(0,0,0,0)$, then system $X_{b_{0}, b_{1}, b_{2}, b_{3}}$ has no limit cycles (see the end of Section 2 in [Dum06]). 


\subsection{Slow-fast Liénard systems (6) in compact sets in the phase space}

Suppose that $K$ is any compact set in the $(\bar{x}, \bar{y})$-plane and fix it. Using [Huz17], in this section we prove that system (6) has at most two limit cycles in $K$, for each fixed $\epsilon \geq 0, \epsilon \sim 0$ and $\left(B_{0}, B_{1}, B_{2}, B_{3}\right) \in \mathbb{S}^{3}$.

In Section 2.2.1 of [Huz17] the following slow-fast systems have been introduced:

$$
\left\{\begin{aligned}
\dot{\bar{x}}= & \bar{y} \\
\dot{\bar{y}}= & -\bar{y} \bar{x}+\epsilon\left(B_{0}+B_{1} \bar{x}+B_{2} \bar{x}^{2}+B_{3} \bar{x}^{3}+\bar{x}^{4}+u \bar{x}^{5} G(u \bar{x}, \lambda)\right) \\
& +\epsilon \bar{y}^{2} H\left(u \bar{x}, u^{2} \bar{y}, \lambda\right),
\end{aligned}\right.
$$

where $\epsilon \geq 0$ is the singular perturbation parameter close to $0,\left(B_{0}, B_{1}, B_{2}, B_{3}\right) \epsilon$ $\mathbb{S}^{3}, u \geq 0$ is close to $0, \lambda$ is kept in an arbitrary compact subset $\Lambda$ of Euclidean space and $G$ and $H$ are arbitrary smooth functions. (System (7) originates from regular codimension 4 saddle-node bifurcations. See Remark 1 for more details.) It has been proved in Section 3 of [Huz17] that for given $G$ and $H$ there exist small numbers $\epsilon_{0}>0$ and $u_{0}>0$ such that system (7) has at most two limit cycles in $K$, for each fixed $\left(\epsilon, u, B_{0}, B_{1}, B_{2}, B_{3}, \lambda\right) \in\left[0, \epsilon_{0}\right] \times\left[0, u_{0}\right] \times \mathbb{S}^{3} \times \Lambda$. When $G \equiv 0$ and $H \equiv 0$, system (7) represents a slow-fast Liénard equation of type (6). Indeed, if we apply $\left(\bar{x}, B_{1}, B_{3}, t\right) \rightarrow\left(-\bar{x},-B_{1},-B_{3},-t\right)$ to $(6)$ we obtain $(7)$ with $G=H \equiv 0$. Thus, we conclude that there exists a small $\epsilon_{0}>0$ such that $(6)$ has at most two limit cycles in $K$, for each fixed $\left(\epsilon, B_{0}, B_{1}, B_{2}, B_{3}\right) \in\left[0, \epsilon_{0}\right] \times \mathbb{S}^{3}$.

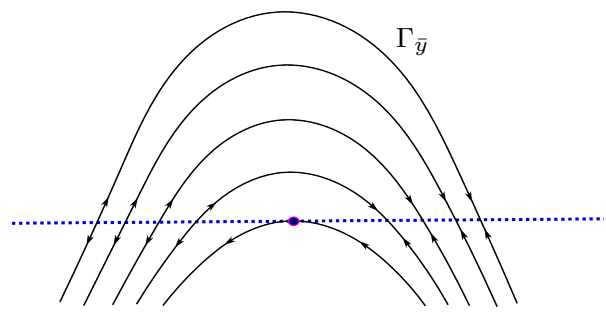

Figure 1: The dynamics of (6) or (7) for $\epsilon=0$, with indication of canard limit periodic sets $\Gamma_{\bar{y}}$ which can produce limit cycles when $\epsilon>0$.

Remark 1. In [Huz17] one studies limit cycles, Hausdorff close to the origin in the phase space, of regular codimension 4 saddle-node bifurcations:

$$
\left\{\begin{aligned}
\dot{x}= & y \\
\dot{y}= & -y x+\left(b_{0}+b_{1} x+b_{2} x^{2}+b_{3} x^{3}+x^{4}+x^{5} G(x, \lambda)\right) \\
& +y^{2} H(x, y, \lambda),
\end{aligned}\right.
$$

where $\left(b_{0}, b_{1}, b_{2}, b_{3}\right) \sim(0,0,0,0), \lambda \in \Lambda$ and $G$ and $H$ are smooth. To show that system (8) produces at most two limit cycles in a small $\left(b_{0}, b_{1}, b_{2}, b_{3}, \lambda\right)$-uniform neighborhood of $(x, y)=(0,0)$ in the phase plane (see Theorem 1.1 of [Huz17]), one uses the rescaling $\left(b_{0}, b_{1}, b_{2}, b_{3}\right)=\left(\epsilon^{4} B_{0}, \epsilon^{3} B_{1}, \epsilon^{2} B_{2}, \epsilon B_{3}\right)$ in the parameter space, like in Section 2.1, and blows up the origin $(x, y, \epsilon)=(0,0,0)$ using the following family blow-up:

$$
(x, y, \epsilon)=\left(u \bar{x}, u^{2} \bar{y}, u \bar{\epsilon}\right), u \geq 0, u \sim 0, \bar{\epsilon} \geq 0,(\bar{x}, \bar{y}, \bar{\epsilon}) \in \mathbb{S}^{2} .
$$


Then one studies system (8) near $(x, y, \epsilon)=(0,0,0)$ in different charts. In the family chart $\{\bar{\epsilon}=1\}$ the blow-up formula becomes $(x, y)=\left(\epsilon \bar{x}, \epsilon^{2} \bar{y}\right)$ and system (8) changes, after dividing by $\epsilon$, into (7), with $u=\epsilon$. The reason why one uses this family blow-up is to reduce the codimension of the system; the codimension of $(7)$ is $\leq 3$ because $\left(B_{0}, B_{1}, B_{2}, B_{3}\right) \in \mathbb{S}^{3}$. Due to the presence of general functions $G$ and $H$ in (7), one studies (7) only in compact subsets $K$ of the $(\bar{x}, \bar{y})$-phase plane (see [Huz17]). There are two different types of limit periodic sets in $K$, at level $\epsilon=0$, that can produce limit cycles in (7) (see Figure 1): the nilpotent contact point $(\bar{x}, \bar{y})=(0,0)$ and the canard limit periodic set $\Gamma_{\bar{y}}$, with $\bar{y}>0$, consisting of the fast orbit of (7) (with $\epsilon=0$ ) passing through the point $(0, \bar{y})$ and the piece of the slow curve $\{\bar{y}=0\}$ between the $\alpha$-limit set and the $\omega$-limit set of the fast orbit. Note that $K$ shrinks to the origin in the original $(x, y)$-phase plane as $\epsilon \rightarrow 0$. To obtain the cyclicity result in (8) in a fixed neighborhood of $(x, y)=(0,0)$ one has to study the phase directional charts $\{\bar{x}= \pm 1\}$ and $\{\bar{y}= \pm 1\}$. For more details see [Huz17].

Since $G=H \equiv 0$ in our paper, we can study system (6) not only in $K$ but also at infinity in the phase plane (see Section 2.3).

\subsection{Slow-fast Liénard systems (6) at infinity in the phase space}

Following [DH99], we can study the dynamics of (6) near infinity on the PoincaréLyapunov disc of type $(2,5)$. Due to the presence of the small parameter $\epsilon>0$, an additional family blow-up in the positive and negative $\bar{x}$-direction is necessary to completely desingularize (6) at infinity. From Sections 2.3.1-2.3.4 it follows that the dynamics of $(6)$ in a $\left(B_{0}, B_{1}, B_{2}, B_{3}, \epsilon\right)$-uniform neighborhood of the infinity is like in Figure 2.

\subsubsection{Transformation of (6) in the positive $\bar{x}$-direction}

We introduce the coordinate change

$$
(\bar{x}, \bar{y})=\left(\frac{1}{\rho^{2}}, \frac{Y}{\rho^{5}}\right)
$$

where $\rho>0, \rho \sim 0$ and $Y$ is kept in a large compact set. In the coordinates $(\rho, Y)$, after multiplication by the positive factor $\rho^{3}$, system (6) can be written as:

$$
\left\{\begin{aligned}
\dot{\rho} & =-\frac{1}{2} \rho Y \\
\dot{Y} & =-\frac{5}{2} Y^{2}-\rho Y-\epsilon\left(B_{0} \rho^{8}+B_{1} \rho^{6}+B_{2} \rho^{4}+B_{3} \rho^{2}+1\right) .
\end{aligned}\right.
$$

When $\rho=0$ and $\epsilon>0$, system (9) has no singularities. When $\rho=\epsilon=0$, the singularity at $Y=0$ of (9) is linearly zero. To desingularize (9) we use the following blow-up at the origin in $(\rho, Y, \epsilon)$-space:

$$
(\rho, Y, \epsilon)=\left(v \bar{\rho}, v \bar{Y}, v^{2} \bar{\epsilon}\right), v \geq 0, v \sim 0, \bar{\epsilon} \geq 0, \bar{\rho} \geq 0,(\bar{\rho}, \bar{Y}, \bar{\epsilon}) \in \mathbb{S}^{2} .
$$

It is convenient to use different charts.

The family chart $\{\bar{\epsilon}=1\}$. System (9) changes, after dividing by $v$, into

$$
\left\{\begin{aligned}
\dot{\bar{\rho}} & =-\frac{1}{2} \bar{\rho} \bar{Y} \\
\dot{\bar{Y}} & =-\frac{5}{2} \bar{Y}^{2}-\bar{\rho} \bar{Y}-1+O\left(v^{2}\right)
\end{aligned}\right.
$$


where $\bar{\rho} \geq 0$ and $(\bar{\rho}, \bar{Y})$ is kept in a large compact set. When $v=0$, system (10) has no singularities.

The phase directional chart $\{\bar{\rho}=1\}$. In the chart $\{\bar{\rho}=1\}$ system (9) becomes, after dividing by $v$,

$$
\left\{\begin{aligned}
\dot{v} & =-\frac{1}{2} v \bar{Y} \\
\dot{\bar{\epsilon}} & =\bar{\epsilon} \\
\dot{\bar{Y}} & =-\bar{Y}-2 \bar{Y}^{2}-\bar{\epsilon}\left(1+O\left(v^{2}\right)\right)
\end{aligned}\right.
$$

where $(v, \bar{\epsilon}) \sim(0,0), v \geq 0, \bar{\epsilon} \geq 0$ and $\bar{Y}$ is kept in a large compact set. When $v=\bar{\epsilon}=0$, system (11) has a hyperbolic saddle at $\bar{Y}=-\frac{1}{2}$ with eigenvalues $\left(\frac{1}{4},-\frac{1}{2}, 1\right)$ and a semi-hyperbolic singularity at $\bar{Y}=0$ with the stable manifold $\{v=\bar{\epsilon}=0\}$ and a two dimensional center manifold transverse to the stable manifold. Center manifolds are given by $\bar{Y}=-\bar{\epsilon}(1+O(v, \bar{\epsilon}))$ and the dynamics inside the center manifolds is given by $\left\{\dot{v}=\frac{1}{2} v \bar{\epsilon}(1+O(v, \bar{\epsilon})), \dot{\bar{\epsilon}}=-\bar{\epsilon}^{2}(1+\right.$ $O(v, \bar{\epsilon}))\}$.

The phase directional chart $\{\bar{Y}=1\}$. In this chart, system (9) changes, after dividing by $v$, into

$$
\left\{\begin{array}{l}
\dot{v}=v\left(-\frac{5}{2}-\bar{\rho}-\bar{\epsilon}\left(1+O\left(v^{2}\right)\right)\right) \\
\dot{\bar{\epsilon}}=-2 \bar{\epsilon}\left(-\frac{5}{2}-\bar{\rho}-\bar{\epsilon}\left(1+O\left(v^{2}\right)\right)\right) \\
\dot{\bar{\rho}}=\bar{\rho}\left(2+\bar{\rho}+\bar{\epsilon}\left(1+O\left(v^{2}\right)\right)\right),
\end{array}\right.
$$

where $(v, \bar{\epsilon}) \sim(0,0), v \geq 0, \bar{\epsilon} \geq 0$ and $\bar{\rho} \geq 0$ is kept in a large compact set. When $v=\bar{\epsilon}=0$, system (12) has a hyperbolic saddle at $\bar{\rho}=0$ with eigenvalues $\left(-\frac{5}{2}, 5,2\right)$.

The phase directional chart $\{\bar{Y}=-1\}$. In this phase directional chart, system (9) changes, after dividing by $v$, into

$$
\left\{\begin{array}{l}
\dot{v}=v\left(\frac{5}{2}-\bar{\rho}+\bar{\epsilon}\left(1+O\left(v^{2}\right)\right)\right) \\
\dot{\bar{\epsilon}}=-2 \bar{\epsilon}\left(\frac{5}{2}-\bar{\rho}+\bar{\epsilon}\left(1+O\left(v^{2}\right)\right)\right) \\
\dot{\bar{\rho}}=\bar{\rho}\left(-2+\bar{\rho}-\bar{\epsilon}\left(1+O\left(v^{2}\right)\right)\right),
\end{array}\right.
$$

where $(v, \bar{\epsilon}) \sim(0,0), v \geq 0, \bar{\epsilon} \geq 0$ and $\bar{\rho} \geq 0$ is kept in a large compact set. Besides the hyperbolic saddle found in the chart $\{\bar{\rho}=1\}$, system (13) has an extra hyperbolic saddle at $(v, \bar{\epsilon}, \bar{\rho})=(0,0,0)$ with eigenvalues $\left(\frac{5}{2},-5,-2\right)$.

\subsubsection{Transformation of (6) in the negative $\bar{x}$-direction}

We define now the coordinate change

$$
(\bar{x}, \bar{y})=\left(\frac{-1}{\rho^{2}}, \frac{Y}{\rho^{5}}\right),
$$

where $\rho>0, \rho \sim 0$ and $Y$ is kept in a large compact set. In the coordinates $(\rho, Y)$, after multiplication by the positive factor $\rho^{3}$, system (6) can be written as:

$$
\left\{\begin{aligned}
\dot{\rho} & =\frac{1}{2} \rho Y \\
\dot{Y} & =\frac{5}{2} Y^{2}+\rho Y-\epsilon\left(B_{0} \rho^{8}-B_{1} \rho^{6}+B_{2} \rho^{4}-B_{3} \rho^{2}+1\right) .
\end{aligned}\right.
$$

When $\rho=0$ and $\epsilon>0$, system (14) has a hyperbolic and attracting node at $Y=-\sqrt{\frac{2 \epsilon}{5}}$ and a hyperbolic and repelling node at $Y=\sqrt{\frac{2 \epsilon}{5}}$. When $\rho=\epsilon=0$, 
the singularity at $Y=0$ of (14) is linearly zero. Like in Section 2.3.1, we blow up the origin $(\rho, Y, \epsilon)=(0,0,0)$ using

$$
(\rho, Y, \epsilon)=\left(v \bar{\rho}, v \bar{Y}, v^{2} \bar{\epsilon}\right), v \geq 0, v \sim 0, \bar{\epsilon} \geq 0, \bar{\rho} \geq 0,(\bar{\rho}, \bar{Y}, \bar{\epsilon}) \in \mathbb{S}^{2} .
$$

The family chart $\{\bar{\epsilon}=1\}$. System (14) changes, after dividing by $v$, into

$$
\left\{\begin{aligned}
\dot{\bar{\rho}} & =\frac{1}{2} \bar{\rho} \bar{Y} \\
\dot{\bar{Y}} & =\frac{5}{2} \bar{Y}^{2}+\bar{\rho} \bar{Y}-1+O\left(v^{2}\right)
\end{aligned}\right.
$$

where $\bar{\rho} \geq 0$ and $(\bar{\rho}, \bar{Y})$ is kept in a large compact set. When $v=0$, system (15) has one hyperbolic and attracting node at $(\bar{\rho}, \bar{Y})=\left(0,-\sqrt{\frac{2}{5}}\right)$ and one hyperbolic and repelling node at $(\bar{\rho}, \bar{Y})=\left(0, \sqrt{\frac{2}{5}}\right)$.

The phase directional chart $\{\bar{\rho}=1\}$. In the chart $\{\bar{\rho}=1\}$ system (14) becomes, after dividing by $v$,

$$
\left\{\begin{aligned}
\dot{v} & =\frac{1}{2} v \bar{Y} \\
\dot{\bar{\epsilon}} & =-\bar{\epsilon} \bar{Y} \\
\dot{\bar{Y}} & =\bar{Y}+2 \bar{Y}^{2}-\bar{\epsilon}\left(1+O\left(v^{2}\right)\right)
\end{aligned}\right.
$$

where $(v, \bar{\epsilon}) \sim(0,0), v \geq 0, \bar{\epsilon} \geq 0$ and $\bar{Y}$ is kept in a large compact set. If $v=\bar{\epsilon}=0$, then system (16) has a hyperbolic saddle at $\bar{Y}=-\frac{1}{2}$ with eigenvalues $\left(-\frac{1}{4}, \frac{1}{2},-1\right)$ and a semi-hyperbolic singularity at $\bar{Y}=0$ with the $\bar{Y}$ axis as the unstable manifold and a two dimensional center manifold transverse to the unstable manifold. Center manifolds can be written as $\bar{Y}=\bar{\epsilon}(1+O(v, \bar{\epsilon}))$, with the following dynamics $\left\{\dot{v}=\frac{1}{2} v \bar{\epsilon}(1+O(v, \bar{\epsilon})), \dot{\bar{\epsilon}}=-\bar{\epsilon}^{2}(1+O(v, \bar{\epsilon}))\right\}$.

The phase directional chart $\{\bar{Y}=1\}$. System (14) changes, after dividing by $v$, into

$$
\left\{\begin{array}{l}
\dot{v}=v\left(\frac{5}{2}+\bar{\rho}-\bar{\epsilon}\left(1+O\left(v^{2}\right)\right)\right) \\
\dot{\bar{\epsilon}}=-2 \bar{\epsilon}\left(\frac{5}{2}+\bar{\rho}-\bar{\epsilon}\left(1+O\left(v^{2}\right)\right)\right) \\
\dot{\bar{\rho}}=\bar{\rho}\left(-2-\bar{\rho}+\bar{\epsilon}\left(1+O\left(v^{2}\right)\right)\right),
\end{array}\right.
$$

where $(v, \bar{\epsilon}) \sim(0,0), v \geq 0, \bar{\epsilon} \geq 0$ and $\bar{\rho} \geq 0$ is kept in a large compact set. System (17) has a hyperbolic saddle at $(v, \bar{\epsilon}, \bar{\rho})=(0,0,0)$ with eigenvalues $\left(\frac{5}{2},-5,-2\right)$.

The phase directional chart $\{\bar{Y}=-1\}$. System (14) changes, after dividing by $v$, into

$$
\left\{\begin{array}{l}
\dot{v}=v\left(-\frac{5}{2}+\bar{\rho}+\bar{\epsilon}\left(1+O\left(v^{2}\right)\right)\right) \\
\dot{\bar{\epsilon}}=-2 \bar{\epsilon}\left(-\frac{5}{2}+\bar{\rho}+\bar{\epsilon}\left(1+O\left(v^{2}\right)\right)\right) \\
\dot{\bar{\rho}}=\bar{\rho}\left(2-\bar{\rho}-\bar{\epsilon}\left(1+O\left(v^{2}\right)\right)\right),
\end{array}\right.
$$

where $(v, \bar{\epsilon}) \sim(0,0), v \geq 0, \bar{\epsilon} \geq 0$ and $\bar{\rho} \geq 0$ is kept in a large compact set. Besides the hyperbolic saddle found in the chart $\{\bar{\rho}=1\}$, we find an extra hyperbolic saddle at $(v, \bar{\epsilon}, \bar{\rho})=(0,0,0)$ of $(18)$ with eigenvalues $\left(-\frac{5}{2}, 5,2\right)$.

\subsubsection{Transformation of (6) in the positive $\bar{y}$-direction}

We introduce the coordinate change

$$
(\bar{x}, \bar{y})=\left(\frac{X}{\rho^{2}}, \frac{1}{\rho^{5}}\right),
$$


where $\rho>0, \rho \sim 0$ and $X$ is kept in a large compact set. In the coordinates $(\rho, X)$, after multiplication by the positive factor $\rho^{3}$, system (6) can be written as:

$$
\left\{\begin{aligned}
\dot{\rho} & =\frac{1}{5} \rho^{2} X+\frac{\epsilon}{5}\left(B_{0} \rho^{9}+B_{1} X \rho^{7}+B_{2} X^{2} \rho^{5}+B_{3} X^{3} \rho^{3}+X^{4} \rho\right) \\
\dot{X} & =\frac{2}{5} X\left(\rho X+\epsilon\left(B_{0} \rho^{8}+B_{1} X \rho^{6}+B_{2} X^{2} \rho^{4}+B_{3} X^{3} \rho^{2}+X^{4}\right)\right)+1 .
\end{aligned}\right.
$$

Besides the singularity in the negative $\bar{x}$-direction there are no extra singularities in the positive $\bar{y}$-direction. The dynamics of (19) points from the left to the right along the $X$-axis.

\subsubsection{Transformation of (6) in the negative $\bar{y}$-direction}

We introduce the transformation

$$
(\bar{x}, \bar{y})=\left(\frac{X}{\rho^{2}}, \frac{-1}{\rho^{5}}\right),
$$

where $\rho>0, \rho \sim 0$ and $X$ is kept in a large compact set. In the coordinates $(\rho, X)$, after multiplication by the positive factor $\rho^{3}$, system (6) can be written as:

$$
\left\{\begin{aligned}
\dot{\rho} & =\frac{1}{5} \rho^{2} X-\frac{\epsilon}{5}\left(B_{0} \rho^{9}+B_{1} X \rho^{7}+B_{2} X^{2} \rho^{5}+B_{3} X^{3} \rho^{3}+X^{4} \rho\right) \\
\dot{X} & =\frac{2}{5} X\left(\rho X-\epsilon\left(B_{0} \rho^{8}+B_{1} X \rho^{6}+B_{2} X^{2} \rho^{4}+B_{3} X^{3} \rho^{2}+X^{4}\right)\right)-1 .
\end{aligned}\right.
$$

Besides the singularity in the negative $\bar{x}$-direction there are no extra singularities in the negative $\bar{y}$-direction. The dynamics of (20) points from the right to the left along the $X$-axis.

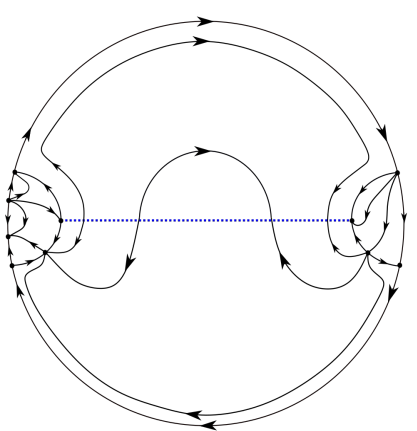

(a)

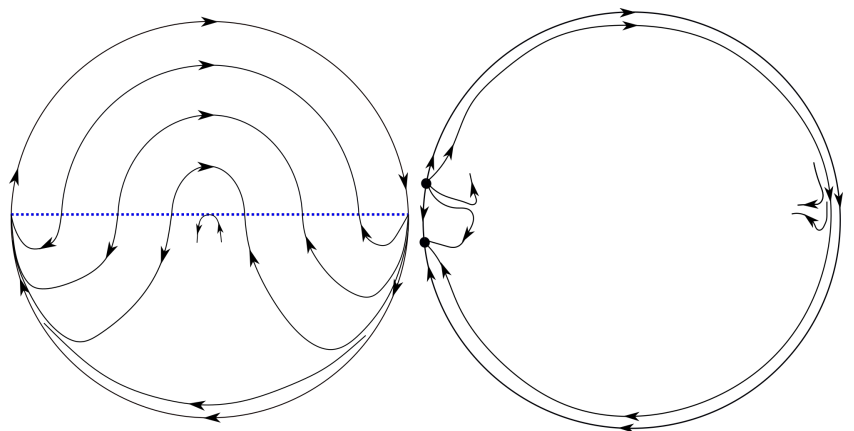

(b)

(c)

Figure 2: Dynamics of (6) near infinity on the Poincaré-Lyapunov disc of type $(2,5)$. (a) The case $\epsilon=0$ with the dynamics on the blow-up locus of the family blow-up at $(\rho, Y, \epsilon)=(0,0,0)$ in the positive $\bar{x}$-direction and the negative $\bar{x}$ direction. (b) The case $\epsilon=0$ after blowing down (a). (c) The case $\epsilon>0$. 


\subsection{Conclusion}

Theorem 1.1 will be proved if we show that there exists a small $\epsilon_{0}>0$ such that system (6) has at most two limit cycles for each fixed $\left(\epsilon, B_{0}, B_{1}, B_{2}, B_{3}\right) \in$ $\left[0, \epsilon_{0}\right] \times \mathbb{S}^{3}$. Following Section 2.2 , this is true in any compact set $K$ in the $(\bar{x}, \bar{y})$-phase plane. Now it suffices to observe that there are no limit periodic sets, containing pieces at infinity, which can generate limit cycles in (6) for $\epsilon>0$. Indeed, Figure 2(c) indicates that any orbit $\mathcal{O}$, containing pieces close to infinity, for $\epsilon>0$ and $\epsilon \sim 0$, cannot be periodic (i.e. closed) because the $\alpha$-limit set of $\mathcal{O}$ is the repelling node at infinity or the $\omega$-limit set of $\mathcal{O}$ is the attracting node at infinity. This completes the proof of Theorem 1.1.

\section{Proof of Theorem 1.2}

Like in Section 2.1, we can suppose that $a_{0}=0$ in (3). We show that there exists a small $\epsilon_{0}>0$ and a small neighborhood $V$ of $\left(b_{0}, b_{1}, b_{2}, b_{3}\right)=(0,0,0,0)$ such that system

$$
\left\{\begin{array}{l}
\dot{x}=y \\
\dot{y}=-y x-\epsilon\left(b_{0}+b_{1} x+b_{2} x^{2}+b_{3} x^{3}+x^{4}\right)
\end{array}\right.
$$

has at most two limit cycles for each fixed $\left(\epsilon, b_{0}, b_{1}, b_{2}, b_{3}\right) \in\left[0, \epsilon_{0}\right] \times V$.

Following Theorem 1.1 of [Huz17], there exists a small $\epsilon_{0}>0$, a small neighborhood $V$ of $\left(b_{0}, b_{1}, b_{2}, b_{3}\right)=(0,0,0,0)$ and a small neighborhood $W$ of $(x, y)=(0,0)$ such that slow-fast codimension 4 saddle-node bifurcations

$$
\left\{\begin{aligned}
\dot{x}= & y \\
\dot{y}= & -y x+\epsilon\left(b_{0}+b_{1} x+b_{2} x^{2}+b_{3} x^{3}+x^{4}+x^{5} G(x, \lambda)\right) \\
& +\epsilon y^{2} H(x, y, \lambda)
\end{aligned}\right.
$$

have at most two limit cycles in $W$, for each $\left(\epsilon, b_{0}, b_{1}, b_{2}, b_{3}, \lambda\right) \in\left[0, \epsilon_{0}\right] \times V \times \Lambda$, with $\Lambda, G$ and $H$ introduced in Section 2.2. If we apply $(x, t) \rightarrow(-x,-t)$ to (21), then (21) becomes a special case of (22). Thus, there exists a small $\epsilon_{0}>0$, a small neighborhood $V$ of $\left(b_{0}, b_{1}, b_{2}, b_{3}\right)=(0,0,0,0)$ and a small neighborhood $W$ of $(x, y)=(0,0)$ such that system $(21)$ has at most two limit cycles in $W$, for each $\left(\epsilon, b_{0}, b_{1}, b_{2}, b_{3}\right) \in\left[0, \epsilon_{0}\right] \times V$.

The slow dynamics of (21) along the slow curve $\{y=0\}$ is given by

$$
x^{\prime}=-\frac{b_{0}+b_{1} x+b_{2} x^{2}+b_{3} x^{3}+x^{4}}{x}, x \neq 0 .
$$

When $\left(b_{0}, b_{1}, b_{2}, b_{3}\right)=(0,0,0,0)$, the slow dynamics $x^{\prime}=-x^{3}$ does not point from the right to the left along the slow curve. This implies that canard cycles $\Gamma_{\bar{y}}$ cannot produce limit cycles in (21), for $\epsilon>0$ and $\epsilon \sim 0$. See Figure 1 .

To find the dynamics of (21) near infinity we use the same Poincaré-Lyapunov compactification as that used in Section 2.3 and we get Figure 2. (Note that (6) and (21) are of same type.) Thus, besides the two limit cycles Hausdorff close to $(x, y)=(0,0)$, there are no extra limit cycles in $(21)$. This completes the proof of Theorem 1.2. 


\section{References}

[Cop89] W. A. Coppel. Some quadratic systems with at most one limit cycle. In Dynamics reported, Vol. 2, volume 2 of Dynam. Report. Ser. Dynam. Systems Appl., pages 61-88. Wiley, Chichester, 1989.

[DH99] F. Dumortier and C. Herssens. Polynomial Liénard equations near infinity. J. Differential Equations, 153(1):1-29, 1999.

[DL96] Freddy Dumortier and Chengzhi Li. On the uniqueness of limit cycles surrounding one or more singularities for Liénard equations. Nonlinearity, 9(6):1489-1500, 1996.

[DL97] Freddy Dumortier and Chengzhi Li. Quadratic Liénard equations with quadratic damping. J. Differential Equations, 139(1):41-59, 1997.

[DMD11] P. De Maesschalck and F. Dumortier. Detectable canard cycles with singular slow dynamics of any order at the turning point. Discrete Contin. Dyn. Syst., 29(1):109-140, 2011.

[DR90] Freddy Dumortier and Christiane Rousseau. Cubic Liénard equations with linear damping. Nonlinearity, 3(4):1015-1039, 1990.

[Dum06] F. Dumortier. Compactification and desingularization of spaces of polynomial Liénard equations. J. Differential Equations, 224(2):296313, 2006.

[Huz17] R. Huzak. Regular and slow-fast codimension 4 saddle-node bifurcations. J. Differential Equations, 262(2):1119-1154, 2017.

[LdMP77] A. Lins, W. de Melo, and C. C. Pugh. On Liénard's equation. In Geometry and topology (Proc. III Latin Amer. School of Math., Inst. Mat. Pura Aplicada CNPq, Rio de Janeiro, 1976), pages 335-357. Lecture Notes in Math., Vol. 597. Springer, Berlin, 1977.

[LL12] C. Li and J. Llibre. Uniqueness of limit cycles for Liénard differential equations of degree four. J. Differential Equations, 252(4):3142-3162, 2012.

[LMT10] J. Llibre, A. C. Mereu, and M. A. Teixeira. Limit cycles of the generalized polynomial Liénard differential equations. Math. Proc. Cambridge Philos. Soc., 148(2):363-383, 2010.

[Pan02] D. Panazzolo. Desingularization of nilpotent singularities in families of planar vector fields. Mem. Amer. Math. Soc., 158(753):viii+108, 2002.

[Rou07] R. Roussarie. Putting a boundary to the space of Liénard equations. Discrete Contin. Dyn. Syst., 17(2):441-448, 2007.

[Sma00] S. Smale. Mathematical problems for the next century. In Mathematics: frontiers and perspectives, pages 271-294. Amer. Math. Soc., Providence, RI, 2000. 
[WJ02] Yu-quan Wang and Zhu-jun Jing. Cubic Lienard equations with quadratic damping. II. Acta Math. Appl. Sin. Engl. Ser., 18(1):103116, 2002. 